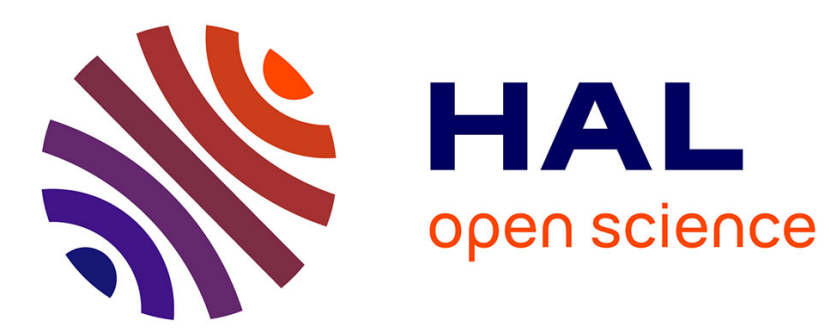

\title{
Event-triggered control of Korteweg-de Vries equation under averaged measurements
}

Wen Kang, Lucie Baudouin, Emilia Fridman

\section{To cite this version:}

Wen Kang, Lucie Baudouin, Emilia Fridman. Event-triggered control of Kortewegde Vries equation under averaged measurements. Automatica, 2021, 123, pp.109315. 10.1016/j.automatica.2020.109315 . hal-02459827v2

\section{HAL Id: hal-02459827 \\ https://hal.science/hal-02459827v2}

Submitted on 25 Sep 2020

HAL is a multi-disciplinary open access archive for the deposit and dissemination of scientific research documents, whether they are published or not. The documents may come from teaching and research institutions in France or abroad, or from public or private research centers.
L'archive ouverte pluridisciplinaire HAL, est destinée au dépôt et à la diffusion de documents scientifiques de niveau recherche, publiés ou non, émanant des établissements d'enseignement et de recherche français ou étrangers, des laboratoires publics ou privés. 


\title{
Event-triggered control of Korteweg-de Vries equation under averaged measurements
}

\author{
Wen Kang $^{\mathrm{a}}$, Lucie Baudouin ${ }^{\mathrm{b}}$, Emilia Fridman ${ }^{\mathrm{c}}$ \\ ${ }^{a}$ School of Automation and Electrical Engineering, University of Science and Technology Beijing, China. \\ b LAAS-CNRS, Université de Toulouse, CNRS, UPS, Toulouse, France. \\ ${ }^{\mathrm{c}}$ Department of Electrical Engineering-Systems, Tel Aviv University, Israel.
}

\begin{abstract}
This work addresses distributed event-triggered control law of 1-D nonlinear Korteweg-de Vries (KdV) equation posed on a bounded domain. Such a system, in a continuous framework, is exponentially stabilizable by a linear state feedback as a source term. Here we consider the situation where the feedback is sampled in time and piecewise averaged in space, and an event-triggering mechanism is designed to maintain stability of this infinite dimensional system. Both well-posedness of the closed-loop system and avoiding the Zeno behaviour issues are addressed. Sufficient LMI-based conditions are constructed to guarantee the regional exponential stability. Numerical examples illustrate the efficiency of the method.
\end{abstract}

Key words: Korteweg-de Vries equation, event-trigger, LMIs.

\section{Introduction}

In fluid mechanics, the Korteweg-de Vries (KdV) equation is a mathematical model of waves on shallow water surfaces in a rectangular channel, equation in which the effects of dispersion, dissipation and nonlinearity are taken into account. When adding a diffusion term, the KdV equation becomes Korteweg-de Vries Burgers (KdVB) equation. The study of $\mathrm{KdV} / \mathrm{KdVB}$ systems has been an active research topic because of its potential applications, see e.g. $[2-4,6,14,16]$. In the field of automatic control, a backstepping approach has been applied in $[4,6,16]$ for the feedback stabilization of KdV equation, and Lyapunov-based arguments have been employed to ensure the stability of the original system under the proposed control law. On the other hand, the survey paper [3] gives a detailed overview of bound-

\footnotetext{
^ This work was supported by Israel Science Foundation (Grant No. 1128/14), National Natural Science Foundation of China (Grant No. 61803026), Fundamental Research Funds for the Central Universities (Grant No. FRF-TP-18032A1), Joint Research Project HetCPS: Ministry of Science \& Technology of Israel and CNRS.

Email addresses: kangwen@amss.ac.cn (Wen Kang), baudouin@laas.fr (Lucie Baudouin), emilia@eng.tau.ac.il (Emilia Fridman).
}

ary controllability and internal stabilization approaches and results for the $\mathrm{KdV}$ equation. One can read in [2] two different approaches (from a Lyapunov functional or from an observability inequality) employed to exponentially stabilize the nonlinear $\mathrm{KdV}$ equation via delayed boundary damping terms.

In [14], distributed control of KdVB system has been suggested under point or averaged localised measurements in space but the proof rely strongly on the presence of a diffusion term that is missing in the $\mathrm{KdV}$ equation. Such distributed control was introduced for heat equation under point [9] and under averaged [10] measurements. In the latter papers, sampled-data control via time-delay approach and Lyapunov-Krasovskii functionals were studied, and the results of $[9,10]$ were extended to event-triggered control in [22]. However, since the Lyapunov-Krasovskii functionals for sampled-data control depend on the state-derivative (see Chapter 7 of [11]), this method cannot be applied to sampled-data control of KdVB equation. So [14] considered the constant input delay case.

To the best of our knowledge, no event-triggered control of KdV equation has been studied yet. The goal of event-triggering mechanism to a sampled control law is to update the control input only at meaningful instants. 
Its drawback, well-known in hybrid systems problematics, could be the exhibition of a Zeno behaviour. This can be summed up as the law bringing an infinite number of updates in a finite amount of time. The present paper aims at contributing to the study of this topic via a Lyapunov approach, where sufficient LMI-based conditions for the closed-loop system with the avoidance of Zeno behaviour will be investigated.

In recent years, event-triggered control systems have been extensively studied (see e.g. $[8,22,24-26]$ ), bringing an important alternative to periodic sampling of control laws. There are many important results on event-triggering mechanisms $[13,25,26]$. In order to reduce out the number of updates, three main event-triggering mechanisms are proposed as follows: continuous event-triggering mechanism (see e.g. [25]), periodic event-triggering mechanism (see e.g. [13]), and event-triggering mechanism with a dwell time (see e.g. $[23,26])$. It is worth pointing out that most works focus on event-triggered control of finite-dimensional systems. However, to the best of our knowledge, there are few papers studying this technique in the infinite-dimensional systems framework (see e.g. $[7,8,22]$ ).

In this work, the main contribution lies in the construction of the event-triggering mechanism and the design of event-triggered control law for nonlinear $\mathrm{KdV}$ equation. It can also be stressed that the LyapunovKrasovskii approaches for sampled-data control design under point/averaged measurements cannot work for $\mathrm{KdV}$ equation. As a by-product, the distributed control via the spatial decomposition (or sampling) for PDEs introduced in [9] and [10] for systems with diffusion terms, is, for the first time, extended to KdV equation that has no such a term. This is achieved thanks to using a $V_{\mu}$ term in Lyapunov functional $V$ defined by (4.5). Such a term is borrowed from [2].

This work addresses the event-triggered control design for KdV system under in domain measurements averaged in space, and for the record, [3] gathers the results for distributed continuous-in-time controller to stabilize the $\mathrm{KdV}$ equation exponentially. Our concern here is then mainly to prove that distributed event-triggered control can still bring, under appropriate assumptions and choice of triggering mechanism, the expected exponential stability. Finally, different from our present work but somehow related to the same area of interest, the exact boundary controllability for the $\mathrm{KdV}$ equation was studied in [20], and [21] is devoted to the design of distributed control for $\mathrm{KdV}$ equation on a periodic domain and to the design of boundary control for $\mathrm{KdV}$ equation on a finite domain.

The remainder of this work is organized as follows. The problem setting is described in Section 2 while Section 3 details the main result of this paper and give some remarks. We suggest finite-dimensional feedback controllers which are distributed on the whole domain or on subdomains under averaged measurements. For both cases, we provide the event-triggering mechanism. Section 4 is devoted to the technical proofs, both of well-posedness of the closed loop system, avoidance of the Zeno behaviour that an event triggering mechanism could introduce, and of the main regional exponential stability theorem. Section 5 contains an extension to distributed on subdomains control and Section 6 presents numerical examples to illustrate the effectiveness of the proposed control strategy. Finally, Section 7 briefly concludes the article.

Notation. For any matrix $P$ in $\mathbb{R}^{n \times n}, P \succ 0$ means that $P$ is symmetric positive definite. For a partitioned matrix, the symbol $*$ stands for symmetric blocks and $I$ is the identity, $\mathbf{0}$ the zero matrix. Using $L^{2}(0, L)$ for the Hilbert space of square integrable scalar functions, one writes $\|u\|_{L^{2}(0, L)}^{2}=\langle u, u\rangle=\int_{0}^{L}|u(x)|^{2} d x$, and we also define the Sobolev spaces $H^{1}(0, L)=\left\{u \in L^{2}(0, L), u^{\prime} \in\right.$ $\left.L^{2}(0, L)\right\}$ and its norm by $\|u\|_{H^{1}(0, L)}^{2}=\|u\|_{L^{2}(0, L)}^{2}+$ $\left\|u^{\prime}\right\|_{L^{2}(0, L)}^{2}, H_{0}^{1}(0, L)=\left\{u \in H^{1}(0, L), u(0)=u(L)=\right.$ $0\}$ where all the derivatives are to be considered in the weak sense. Finally, $L^{\infty}(0, L)$ denotes the space of essentially bounded function. For a function $y$ of several variables, the partial derivative with respect to a variable $\xi$ is denoted $\partial_{\xi} y=\frac{\partial y}{\partial \xi}$.

\section{Preliminaries and problem formulation}

\subsection{State feedback control of a nonlinear $K d V$ equation}

Before proceeding to our problem's setting, let us explain the essential idea of the Lyapunov-based state feedback control for KdV equation. Consider the initial and boundary value problem

$$
\left\{\begin{array}{rr}
\partial_{t} z+z \partial_{x} z+\partial_{x} z+\nu \partial_{x x x} z-\lambda z=f(x, t) & \forall x \in(0, L), t \geq 0, \\
z(0, t)=z(L, t)=0, \partial_{x} z(L, t)=0, & \forall t \geq 0 \\
z(x, 0)=z^{0}(x), & \forall x \in(0, L),
\end{array}\right.
$$

with the initial state $z^{0} \in L^{2}(0, L)$ and the source input $f \in L^{1}\left(0, T ; L^{2}(0, L)\right)$, where $\nu>0, \lambda \geq 0$, and $z=z(x, t)$ is the state of the nonlinear $\mathrm{KdV}$ equation. For $\lambda>0$, the open-loop system may be unstable (see the example below). Note that destabilizing $\lambda>0$ was considered in [27]. Also, $\lambda>0$ may stand for the desired decay rate achieved after stabilization of (2.1) with $\lambda=0$ (see Remark 3 below).

By selecting the control law

$$
f(x, t)=-K z(x, t), \quad K>\lambda,
$$


one obtains a closed-loop system that is globally exponentially stable, as it will be shown later. In this article, we would like to address the question of the robustness of this stability with respect to the presence of both an event triggering in time and a localized averaging in space of the feedback control law. Noticing that we cannot really apply infinite dimensional feedback control law, we will consider here a finite dimensional approximation of (2.2) that still stabilizes the system (see Section 3 below).

More precisely, we will consider that the control law will be implemented in such a way that for all $x \in(0, L)$, for all $t \in\left[t_{k}, t_{k+1}\right)$,

$$
f(x, t)=-K \sum_{j=1}^{N} \bar{z}_{j}\left(t_{k}\right) \mathbb{1}_{\Omega_{j}}(x), \quad K>\lambda,
$$

where the sampling times $t_{k}$ are following an appropriate event trigger law to be given later, while $\left\{\mathbb{1}_{\Omega_{j}}\right\}_{j}$ are the characteristic functions of the intervals $\left\{\Omega_{j}\right\}_{j}$ covering $(0, L)$, and $\bar{z}_{j}(t)=\frac{1}{\left|\Omega_{j}\right|} \int_{\Omega_{j}} z(x, t) d x$. We will also consider the case that the event-triggered controller does not cover the whole domain $[0, L]$, which is distributed on some parts of subdomains (see (5.1) in Section 5 below).

\subsection{Well-posedness and exponential stabilization result under (2.2)}

The proof of existence and regularity of solutions for the $\mathrm{KdV}$ equation has been investigated in many references, in particular in the field of controllability studies and even if several results rely on the smallness of the initial and source data (e.g. $[3,6]$ ), one can find in [5] the proof of the following general result:

Lemma 1. For any $T>0, L>0$, if $z^{0} \in L^{2}(0, L)$ and $f \in L^{1}\left(0, T ; L^{2}(0, L)\right)$, then the Cauchy problem (2.1) is well posed in the space $C\left([0, T] ; L^{2}(0, L)\right) \cap$ $L^{2}\left(0, T ; H^{1}(0, L)\right)$, meaning that there exists a unique solution $z$ to the system (2.1) that satisfies, for a constant $c=c(T, L)>0$,

$$
\begin{aligned}
\|z\|_{L^{\infty}\left(0, T ; L^{2}(0, L)\right)}+\|z\|_{L^{2}\left(0, T ; H^{1}(0, L)\right)} \\
\leq c\left\|z^{0}\right\|_{L^{2}(0, L)}+c\|f\|_{L^{1}\left(0, T ; L^{2}(0, L)\right)}
\end{aligned}
$$

The proof of this lemma is detailed in [5] and relies on a fixed point argument for the small time wellposedness (as also referenced and described in [3]) of the problem, that allows to handle the non-linearity $z \partial_{x} z$, and on clever a priori estimates of the local solution to extend arbitrarily the time frame and get a global existence and regularity result.

The proof of the well-posedness of the closed-loop system (2.1)-(2.2) stems from the same arguments and is not detailed here. Besides, it is easy to prove its exponential stability, stated here:

Lemma 2. Let $L>0, T>0, K>\lambda$ and $z^{0} \in L^{2}(0, L)$. The closed-loop KdV system (2.1)-(2.2) is exponentially stable in the sense that

$$
\|z(\cdot, t)\|_{L^{2}(0, L)}^{2} \leq e^{-2(K-\lambda) t}\left\|z^{0}\right\|_{L^{2}(0, L)}^{2}, \forall t \geq 0 .
$$

Indeed : define the energy (that will act as a Lyapunov functional) of the solution of a $\mathrm{KdV}$ equation by

$$
E(t)=\|z(\cdot, t)\|_{L^{2}(0, L)}^{2}, \quad \forall t \geq 0 .
$$

Taking the time derivative of $E(t)$ along (2.1)-(2.2), we have, for any $t \geq 0$,

$$
\begin{aligned}
\dot{E}(t) & \leq-2(K-\lambda) \int_{0}^{L}|z(x, t)|^{2} d x-\nu\left|\partial_{x} z(0, t)\right|^{2} \\
& \leq-2(K-\lambda) E(t)
\end{aligned}
$$

implying $E(t) \leq e^{-2(K-\lambda) t} E(0), \forall t \geq 0$.

Furthermore, [3] gathers several internal stabilization results for nonlinear $\mathrm{KdV}$ equations, and specifically, the stabilization through a localized distributed internal damping $f(x, t)=-a(x) z(x, t)$ with $a \in L^{\infty}(0, L)$ such that $a(\cdot) \geq a_{0}>\lambda$ in some subdomain $\omega$ of $(0, L)$, is actually also true, see e.g. $[17,19]$. However, in our study, we focus on the case described by $(2.3)$.

As already mentioned before, in this paper we will use a Lyapunov approach to deal with an event-triggered control of the $\mathrm{KdV}$ equation under averaged measurements. The next section is devoted to the description of our technical setting.

\section{Problem formulation and main result}

We consider the following closed-loop KdV system:

$$
\left\{\begin{array}{r}
\partial_{t} z+z \partial_{x} z+\partial_{x} z+\nu \partial_{x x x} z-\lambda z \\
=-K \sum_{j=1}^{N} \bar{z}_{j}\left(t_{k}\right) \mathbb{1}_{\Omega_{j}}(x) \\
\operatorname{in}(0, L) \times\left[t_{k}, t_{k+1}\right), \quad k \in \mathbb{N}, \\
z(0, t)=z(L, t)=0, \partial_{x} z(L, t)=0, \forall t \geq 0, \\
z(x, 0)=z^{0}(x), \quad \forall x \in(0, L) .
\end{array}\right.
$$

where the chosen control law for (2.1) is (2.3),

$$
\bar{z}_{j}\left(t_{k}\right)=\frac{1}{\left|\Omega_{j}\right|} \int_{\Omega_{j}} z\left(x, t_{k}\right) d x .
$$


This closed-loop system is defined under the following assumptions:

- Space averaging: As in $[1,9,10,15]$, we assume that the points $0=x_{0}<x_{1}<\cdots<x_{N}=L$ divide the interval $[0, L]$ into $N$ intervals $\Omega_{j}=\left[x_{j-1}, x_{j}\right)$ covering it all. The width of each sub-interval is supposed to be upper bounded by some constant: $0<x_{j}-x_{j-1}=$ $\left|\Omega_{j}\right| \leq \Delta$ and as expected, the characteristic functions $\mathbb{1}_{\Omega_{j}}(\bar{x})$ are such that

$$
\left\{\begin{array}{l}
\mathbb{1}_{\Omega_{j}}(x)=0, x \notin \Omega_{j}, \\
\mathbb{1}_{\Omega_{j}}(x)=1, \text { otherwise },
\end{array} \quad j=1, \cdots, N .\right.
$$

- Time sampling: The update instants satisfy $0=t_{0}<$ $t_{1}<\cdots<t_{k}<t_{k+1}, \lim _{k \rightarrow \infty} t_{k}=\infty$. We define the event trigger mechanism by the law

$$
\begin{aligned}
& t_{k+1}=\inf \left\{t \geq t_{k}\right. \text { such that } \\
& \left.\left\|z(\cdot, t)-z\left(\cdot, t_{k}\right)\right\|_{L^{2}(0, L)}^{2} \geq \gamma E(t)+\gamma_{0} E(0) e^{-2 \theta t}\right\}
\end{aligned}
$$

where the energy $E$ is defined by (2.4) as the $L^{2}(0, L)$-norm of the state, and $\gamma, \gamma_{0}$ and $\theta$ are positive constants to be determined.

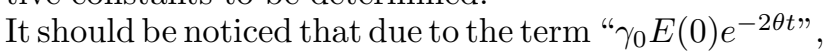
here no dwell time is needed to be defined.

- Though the feedback is of finite dimension, both $t_{k}$ and $\Delta$ depend on the initial data. The larger initial data is, the smaller $t_{k}$ and $\Delta$ need to be.

Our main objective is to design a regionally stabilizing event-trigger controller

$$
u_{j}(t)=-K \bar{z}_{j}\left(t_{k}\right) \mathbb{1}_{\left[t_{k}, t_{k+1}\right)}(t)
$$

that has a control gain $K>\lambda$ to be determined later. In other words, we aim at deriving sufficient conditions for regional exponential stability of the closed-loop system (3.1) and to find a bound on the domain of attraction.

Theorem 1. Let $L>0, T>0$. Given a desired decay rate $\delta>0$, a control gain $K>\lambda+\delta$, a length bound $\Delta>0$, and positive tuning parameters $\lambda_{0}, R, \theta>\delta$, $\gamma_{0}>0$, assume that there exist positive scalars $\mu, \lambda_{1}, \lambda_{2}$, $\gamma$, and $\Gamma$ that solve the following optimization problem:

$$
\begin{gathered}
\min \Gamma \text { subject to } \\
-3 \mu \nu+\lambda_{1}+\lambda_{2}+\frac{2}{3} \mu R L \sqrt{L}<0, \\
\Phi=\left[\begin{array}{ccc}
\phi_{11} & K(1+\mu L) & K(1+\mu L) \\
* & -\lambda_{2} \frac{\pi^{2}}{\Delta^{2}} & 0 \\
* & * & -\lambda_{0}
\end{array}\right] \prec 0,
\end{gathered}
$$

$$
(1+\mu L)\left(1+\frac{\lambda_{0} \gamma_{0}}{2(\theta-\delta)}\right)<R^{2} \Gamma
$$

where

$$
\phi_{11}=-2 K+2 \lambda+\mu+\lambda_{0} \gamma-\lambda_{1} \frac{\pi^{2}}{L^{2}}+2 \delta .
$$

Then for any initial function $z^{0} \in L^{2}(0, L)$ satisfying $\left\|z^{0}\right\|_{L^{2}(0, L)}<\frac{1}{\sqrt{\Gamma}}$, the closed-loop system (3.1) under the event-triggering mechanism (3.4) is exponentially stable:

$$
E(t) \leq\left(1+\frac{\lambda_{0} \gamma_{0}}{2(\theta-\delta)}\right)(1+\mu L) E(0) e^{-2 \delta t}
$$

for all $t \geq 0$. Moreover, if the above LMIs hold with $\delta=0$, then the closed-loop system is exponentially stable with a small enough decay rate.

Remark 1. One could wish here that we do not make the assumption $K \geq \lambda+\delta$ on the gain we need to apply to stabilize our system, but we shall recall that the decay rate of the exponential stability of the system with continuous feedback law $-K z$ is excatly $\delta=K-\lambda$ (Lemma 2) so that it's not reasonable to expect better when applying an approximated feedback law as we do.

Remark 2. If $\gamma$ and $\gamma_{0}$ are small enough, then the event-triggering mechanism (3.4) gets more sensitive to the output change and transmits the signals more often, what makes the control more similar to the stabilizing continuous-time controller.

Remark 3. Consider (3.1) with $\lambda=0$

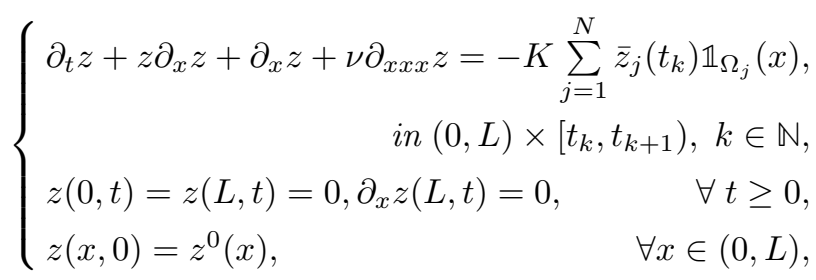

where $\bar{z}_{j}\left(t_{k}\right)$ is given by (3.2).

Let $\bar{z}=e^{\lambda t} z$. It is easy to see that $\bar{z}$ is governed by

$$
\left\{\begin{array}{l}
\partial_{t} \bar{z}+e^{-\lambda t} \bar{z} \partial_{x} \bar{z}+\partial_{x} \bar{z}+\nu \partial_{x x x} \bar{z}-\lambda \bar{z} \\
=-K \sum_{j=1}^{N} \hat{z}_{j}\left(t_{k}\right) \mathbb{1}_{\Omega_{j}}(x), \\
\quad i n(0, L) \times\left[t_{k}, t_{k+1}\right), \quad k \in \mathbb{N}, \\
\bar{z}(0, t)=\bar{z}(L, t)=0, \partial_{x} \bar{z}(L, t)=0, \forall t \geq 0, \\
\bar{z}(x, 0)=z^{0}(x), \quad \forall x \in(0, L),
\end{array}\right.
$$

where $\hat{z}_{j}\left(t_{k}\right)=\frac{1}{\left|\Omega_{j}\right|} \int_{\Omega_{j}} \bar{z}\left(x, t_{k}\right) d x$.

From the proof of Theorem 1, it follows that LMIs of this Theorem guarantee stability of (3.11) since the nonlinear term " $e^{-\lambda t} \bar{z}_{\bar{z}}$ " with the multiplier $e^{-\lambda t} \leq 1$ will not 
change the proof of stability. Hence, if the LMI conditions of Theorem 1 hold with $\delta=0$, then the decay rate $\lambda$ of original system (3.10) can be guaranteed since $z=e^{-\lambda t} \bar{z}$.

\section{Technical proofs}

\subsection{Well-posedness of the controlled system and avoid-} ance of Zeno behaviour

From Lemma 1, the following well-posedness result can be obtained by an induction approach.

Proposition 1. Let $L>0, T>0$ and assume that $z^{0} \in L^{2}(0, L)$. Then system (3.1) under the event triggering law (3.4) has a unique solution $z$ satisfying $z \in$ $C\left(0, T ; L^{2}(0, L)\right) \cap L^{2}\left(0, T ; H^{1}(0, L)\right)$. Furthermore, the Zeno phenomenon is avoided.

Proof. In oder to prove the existence, uniqueness and regularity of the solution, we proceed by induction.

(i) Initialization. On the first time interval, (3.1) reads

$$
\left\{\begin{array}{rrr}
\partial_{t} z+z \partial_{x} z+\partial_{x} z+\nu \partial_{x x x} z-\lambda z=-K \sum_{j=1}^{N} \bar{z}_{j}^{0} \mathbb{1}_{\Omega_{j}}(x), & \forall x \in(0, L), & t \in\left[0, t_{1}\right), \\
z(0, t)=z(L, t)=0, \partial_{x} z(L, t)=0, & \forall t \geq 0, \\
z(x, 0)=z^{0}(x), & \forall x \in(0, L),
\end{array}\right.
$$

where $\bar{z}_{j}^{0}=\bar{z}_{j}(0)=\frac{1}{\left|\Omega_{j}\right|} \int_{\Omega_{j}} z^{0}(x) d x$, and $K>\lambda$. This is a nonlinear $\mathrm{KdV}$ equation with initial data $z^{0} \in L^{2}(0, L)$ and source term $f=-K \sum_{j=1}^{N} \bar{z}_{j}^{0} \mathbb{1}_{\Omega_{j}} \in L^{1}\left(0, t_{1} ; L^{2}(0, L)\right)$. Lemma 1 allows to conclude that there exists a unique solution $z \in C\left(\left[0, t_{1}\right] ; L^{2}(0, L)\right) \cap L^{2}\left(0, t_{1} ; H_{0}^{1}(0, L)\right)$ to the latter system.

(ii) Heredity. Let us only highlight that the previously obtained solution satisfies $z\left(t_{1}\right) \in L^{2}(0, L)$ so that system (3.1) considered on the next time interval $\left[t_{1}, t_{2}\right)$ has an initial condition $z\left(t_{1}\right) \in L^{2}(0, L)$ and a source term $-K \sum_{j=1}^{N} \bar{z}_{j}^{1} \mathbb{1}_{\Omega_{j}} \in L^{1}\left(t_{1}, t_{2} ; L^{2}(0, L)\right.$ where $\bar{z}_{j}^{1}=\bar{z}_{j}\left(t_{1}\right)$. Therefore, the same argument using Lemma 1 holds again and the heredity is proved similarly at any step $k \in \mathbb{N}$.

(iii) Conclusion. By induction, for any $k \in \mathbb{N}$, $z \in C\left(\left[t_{k}, t_{k+1}\right] ; L^{2}(0, L)\right) \cap L^{2}\left(t_{k}, t_{k+1} ; H^{1}(0, L)\right)$. Therefore, from the extension by continuity at the instants $t_{k}$, one can conclude that (3.1) has a unique solution $z \in C\left([0, T] ; L^{2}(0, L)\right) \cap L^{2}\left(0, T ; H^{1}(0, L)\right)$.

(iv) Convergence. The solution will never blow up before $T$ as a contrary of the Zeno behaviour (i.e. $\exists t_{k}>T$ ).
Now we aim at showing that the event-triggering mechanism (3.4) rules out the Zeno behaviour, where an infinite number of updates may occur in a finite amount of time. It is actually sufficient to show that for a given $T>0$, there exists $\tau^{*}>0$ such that all the sampling instants $t_{k} \leq T$ complying to (3.4) satisfy $t_{k+1}-t_{k} \geq \tau^{*}$. Let us denote by $e_{k}$ the deviation from the continuous time position: for any $x \in[0, L]$ and $t \in(0, T)$, there exists $k \in \mathbb{N}$ such that $t \in\left[t_{k}, t_{k+1}\right)$, and we set

$$
e_{k}(x, t) \triangleq z(x, t)-z\left(x, t_{k}\right)
$$

Since the solution of closed-loop system (3.1) satisfies $z \in C\left([0, T] ; L^{2}(0, L)\right)$ and $[0, T]$ is a compact set, this error function $e_{k}$ is uniformly continuous in time with values in $L^{2}(0, L)$. This means that for any $\epsilon>0$ there exists $\tau^{*}>0$ such that for all $t, s \in[0, T]$, if $|t-s|<\tau^{*}$ then we have $\left\|e_{k}(\cdot, t)-e_{k}(\cdot, s)\right\|_{L^{2}(0, L)}<\epsilon$.

Thus, the following reasoning by contraposition holds: $\forall \epsilon>0, \exists \tau^{*}>0, \forall t, s \in[0, T]$,

$$
\left\|e_{k}(\cdot, t)-e_{k}(\cdot, s)\right\|_{L^{2}(0, L)} \geq \epsilon \Longrightarrow|t-s| \geq \tau^{*}
$$

Since $e_{k}\left(t_{k}\right)=0$, we have

$$
\left\|e_{k}\left(\cdot, t_{k+1}\right)-e_{k}\left(\cdot, t_{k}\right)\right\|_{L^{2}(0, L)}=\left\|e_{k}\left(\cdot, t_{k+1}\right)\right\|_{L^{2}(0, L)} .
$$

Next the substitution $t \rightarrow t_{k+1}$ and $s \rightarrow t_{k}$ into (4.2), together with the definition of $t_{k+1}$ in (3.4), leads to

$$
\begin{aligned}
\left\|e_{k}\left(\cdot, t_{k+1}\right)\right\|_{L^{2}(0, L)}^{2} & \geq \gamma E\left(t_{k+1}\right)+\gamma_{0} E(0) e^{-2 \theta t_{k+1}} \\
& \geq \gamma_{0}\left\|z^{0}\right\|_{L^{2}(0, L)}^{2} e^{-2 \theta T}
\end{aligned}
$$

implying that $\left|t_{k+1}-t_{k}\right| \geq \tau^{*}$. Indeed, given $z^{0} \neq 0$, we choose $\epsilon=\sqrt{\gamma_{0}\left\|z^{0}\right\|_{L^{2}(0, L)}^{2} e^{-2 \theta T}}$ so that there exists $\tau^{*}>0$, depending on $z^{0}, \theta, \gamma_{0}, \gamma$ and $T$ for which for any $k$ such that $t_{k}, t_{k+1} \in[0, T]$, one has $t_{k+1}-t_{k} \geq \tau^{*}$, so that the Zeno behaviour is avoided.

\subsection{Regional stability analysis}

Now we focus on the regional stability analysis of the closed-loop system and prove Theorem 1. Let us mention two things.

On the one hand, the event-triggering mechanism (3.4) yields that the event-triggering error function is bounded on each time sub-interval as follows: $\forall t \in\left[t_{k}, t_{k+1}\right)$

$$
\left\|e_{k}(\cdot, t)\right\|_{L^{2}(0, L)}^{2} \leq \gamma E(t)+\gamma_{0} E(0) e^{-2 \theta t} .
$$


On the other hand, $f$ defined by (2.3) can be rewritten as

$$
\begin{array}{r}
f(x, t)=-K \sum_{j=1}^{N} \mathbb{1}_{\Omega_{j}}(x)\left[z(x, t)-f_{j}(x, t)-\rho_{j}(t)\right], \\
\forall x \in[0, L], \forall t \in\left[t_{k}, t_{k+1}\right), \forall k \in \mathbb{N}
\end{array}
$$

where

$$
\begin{aligned}
& f_{j}(x, t)=z(x, t)-\bar{z}_{j}(t)=z(x, t)-\frac{1}{\left|\Omega_{j}\right|} \int_{\Omega_{j}} z(x, t) d x \\
& \rho_{j}(t)=\bar{z}_{j}(t)-\bar{z}_{j}\left(t_{k}\right)=\frac{1}{\left|\Omega_{j}\right|} \int_{\Omega_{j}} e_{k}(x, t) d x
\end{aligned}
$$

\section{Proof of Theorem 1}

Writing $V_{\mu}(t)=\mu \int_{0}^{L} x|z(x, t)|^{2} d x$ with $\mu>0$, we define the following functional (see [2]):

$V(t) \triangleq E(t)+V_{\mu}(t)=\int_{0}^{L}|z(x, t)|^{2} d x+\mu \int_{0}^{L} x|z(x, t)|^{2} d x$.

First, this Lyapunov functional candidate $V(t)$ is equivalent to the energy of the system $E(t)$ in the sense that

$$
E(t) \leq V(t) \leq(1+\mu L) E(t)
$$

Then, let us estimate its time derivative. For $t \in$ $\left[t_{k}, t_{k+1}\right)$, substituting (4.4) into (3.1) and differentiating $V(t)$ along (3.1), one gets

$$
\begin{aligned}
& \dot{V}(t)=\dot{E}(t)+\dot{V}_{\mu}(t) \\
& =2 \int_{0}^{L} z(x, t) \partial_{t} z(x, t) d x+2 \mu \int_{0}^{L} x z(x, t) \partial_{t} z(x, t) d x \\
& =2 \int_{0}^{L}(1+\mu x) z(x, t)\left[-\nu \partial_{x x x} z(x, t)-z(x, t) \partial_{x} z(x, t)\right. \\
& \left.\quad-\partial_{x} z(x, t)+\lambda z(x, t)-K z(x, t)\right] d x \\
& +2 K \sum_{j=1}^{N} \int_{\Omega_{j}}(1+\mu x) z(x, t)\left[f_{j}(x, t)+\rho_{j}(t)\right] d x .
\end{aligned}
$$

Hence,

$$
\begin{aligned}
\dot{V}(t)= & -\nu\left|\partial_{x} z(0, t)\right|^{2}-3 \mu \nu \int_{0}^{L}\left|\partial_{x} z(x, t)\right|^{2} d x \\
& +\mu \int_{0}^{L}|z(x, t)|^{2} d x+\frac{2}{3} \mu \int_{0}^{L} z^{3}(x, t) d x \\
& -2(K-\lambda) \int_{0}^{L}(1+\mu x)|z(x, t)|^{2} d x \\
& +2 K \sum_{j=1}^{N} \int_{\Omega_{j}}(1+\mu x) z(x, t)\left[f_{j}(x, t)+\rho_{j}(t)\right] d x .
\end{aligned}
$$

Using (4.3), for any $\lambda_{0}>0$ we can deduce that

$$
\begin{aligned}
& \dot{V}(t) \leq \dot{V}(t)+\lambda_{0}\left[\gamma E(t)+\gamma_{0} E(0) e^{-2 \theta t}-\left\|e_{k}(\cdot, t)\right\|_{L^{2}(0, L)}^{2}\right] \\
& \leq-3 \mu \nu \int_{0}^{L}\left|\partial_{x} z(x, t)\right|^{2} d x-\left(2 K-\mu-\lambda_{0} \gamma-2 \lambda\right) \int_{0}^{L}|z(x, t)|^{2} d x \\
& +\frac{2}{3} \mu \int_{0}^{L} z^{3}(x, t) d x-(2 K-2 \lambda) \mu \int_{0}^{L} x|z(x, t)|^{2} d x \\
& +2 K \sum_{j=1}^{N} \int_{\Omega_{j}}(1+\mu x) z(x, t)\left[f_{j}(x, t)+\rho_{j}(t)\right] d x \\
& +\lambda_{0} \gamma_{0} E(0) e^{-2 \theta t}-\lambda_{0}\left\|e_{k}(\cdot, t)\right\|_{L^{2}(0, L)}^{2}
\end{aligned}
$$

Several estimates can now be obtained to deal with each of these terms and bring this into a quadratic form. First, Cauchy-Schwarz inequality and Sobolev's inequality (see Lemma A.3) leads to

$$
\begin{array}{r}
\int_{0}^{L} z^{3}(x, t) d x \leq\|z(\cdot, t)\|_{L^{\infty}(0, L)}^{2} \int_{0}^{L}|z(x, t)| d x \\
\leq L \sqrt{L}\left\|\partial_{x} z(\cdot, t)\right\|_{L^{2}(0, L)}^{2}\|z(\cdot, t)\|_{L^{2}(0, L)}
\end{array}
$$

Then from Lemma A.4, Wirtinger's inequality yields

$$
\lambda_{1}\left[\left\|\partial_{x} z(\cdot, t)\right\|_{L^{2}(0, L)}^{2}-\frac{\pi^{2}}{L^{2}}\|z(\cdot, t)\|_{L^{2}(0, L)}^{2}\right] \geq 0 .
$$

for any $\lambda_{1}>0$.

Moreover, since $\int_{\Omega_{j}} f_{j}(x, t) d x=0$, from Lemma A.5, Poincaré's inequality rewrites

$$
\left\|f_{j}(\cdot, t)\right\|_{L^{2}\left(\Omega_{j}\right)}^{2} \leq \frac{\Delta^{2}}{\pi^{2}}\left\|\partial_{x} z(\cdot, t)\right\|_{L^{2}\left(\Omega_{j}\right)}^{2},
$$

bringing for any $\lambda_{2}>0$

$$
\lambda_{2} \sum_{j=1}^{N}\left[\left\|\partial_{x} z(\cdot, t)\right\|_{L^{2}\left(\Omega_{j}\right)}^{2}-\frac{\pi^{2}}{\Delta^{2}}\left\|f_{j}(\cdot, t)\right\|_{L^{2}\left(\Omega_{j}\right)}^{2}\right] \geq 0 .
$$

Applying the Cauchy-Schwarz inequality, we obtain

$$
\begin{aligned}
& \sum_{j=1}^{N} \int_{\Omega_{j}} \rho_{j}^{2}(t)=\sum_{j=1}^{N} \rho_{j}^{2}(t)\left|\Omega_{j}\right| \leq \sum_{j=1}^{N} \frac{1}{\left|\Omega_{j}\right|}\left(\int_{\Omega_{j}} e_{k}(x, t) d x\right)^{2} \\
& \leq \sum_{j=1}^{N} \int_{\Omega_{j}} e_{k}^{2}(x, t) d x=\int_{0}^{L} e_{k}^{2}(x, t) d x
\end{aligned}
$$

Hence,

$$
\lambda_{0}\left[\left\|e_{k}(\cdot, t)\right\|_{L^{2}(0, L)}^{2}-\sum_{j=1}^{N} \int_{\Omega_{j}} \rho_{j}^{2}(t)\right] \geq 0 .
$$

Set $\eta(x, t)=\operatorname{col}\left\{z(x, t), f_{j}(x, t), \rho_{j}(t)\right\}$. Substituting (4.8) and (4.11) into (4.7), and adding (4.9), (4.10) and 
(4.12) to $\dot{V}(t)$, we obtain

$$
\begin{aligned}
& \dot{V}(t)+2 \delta V(t) \leq \sum_{j=1}^{N} \int_{\Omega_{j}} \eta(x, t)^{\top} \Phi(x) \eta(x, t) \\
& -\left(3 \mu \nu-\lambda_{1}-\lambda_{2}-\frac{2 \mu}{3} L \sqrt{L}\|z(\cdot, t)\|_{L^{2}(0, L)}\right)\left\|\partial_{x} z(\cdot, t)\right\|_{L^{2}(0, L)}^{2} \\
& -2(K-\lambda-\delta) \mu \int_{0}^{L} x|z(x, t)|^{2} d x+\lambda_{0} \gamma_{0} E(0) e^{-2 \theta t},
\end{aligned}
$$

where

$$
\Phi(x)=\left[\begin{array}{ccc}
\phi_{11} & K(1+\mu x) & K(1+\mu x) \\
* & -\lambda_{2} \frac{\pi^{2}}{\Delta^{2}} & 0 \\
* & * & -\lambda_{0}
\end{array}\right]
$$

and $\phi_{11}=-2 K+2 \lambda+\mu+\lambda_{0} \gamma-\lambda_{1} \frac{\pi^{2}}{L^{2}}+2 \delta$ as in (3.8). Applying Schur complement theorem [11], one gets that $\Phi(x) \prec 0$ is equivalent to

$$
\phi_{11}+K^{2}(1+\mu x)^{2}\left(\frac{\Delta^{2}}{\lambda_{2} \pi^{2}}+\lambda_{0}^{-1}\right)<0,
$$

that also writes

$$
\begin{aligned}
& -2 K+2 \lambda+\mu+\lambda_{0} \gamma-\lambda_{1} \frac{\pi^{2}}{L^{2}} \\
& +2 \delta+K^{2}(1+\mu x)^{2}\left(\frac{\Delta^{2}}{\lambda_{2} \pi^{2}}+\lambda_{0}^{-1}\right)<0
\end{aligned}
$$

Since we need that property for all $x \in[0, L]$, and since we have $1 \leq(1+\mu x)^{2} \leq(1+\mu L)^{2}$, then it proves

$$
\Phi(L) \prec 0 \quad \Longrightarrow \quad \Phi(x) \prec 0, \forall x \in[0, L] .
$$

Hence, denoting $\Phi=\Phi(L)$ so that (3.6) holds, we have proved that

$$
\sum_{j=1}^{N} \int_{\Omega_{j}} \eta(x, t)^{\top} \Phi(x) \eta(x, t) \leq 0 .
$$

A final step as to be performed to handle the nonquadratic estimate (4.8). Let us first assume that

$$
\|z(\cdot, t)\|_{L^{2}(0, L)}<R, \forall t \geq 0
$$

Under assumptions (3.5)-(3.6) and (4.16), from (4.13) and (4.15) and choosing $K>\lambda+\delta$, we obtain

$\dot{V}(t)+2 \delta V(t) \leq \lambda_{0} \gamma_{0} E(0) e^{-2 \theta t} \leq \lambda_{0} \gamma_{0} V(0) e^{-2 \theta t}, \forall t \geq 0$
Now let $\theta>\delta$. Then, for all $t \geq 0$ we can write

$$
\begin{aligned}
& V(t) \leq e^{-2 \delta t} V(0)+\lambda_{0} \gamma_{0} e^{-2 \delta t} V(0) \int_{0}^{t} e^{-2(\theta-\delta) s} d s \\
& \leq e^{-2 \delta t} V(0)+\frac{\lambda_{0} \gamma_{0} V(0)}{2(\theta-\delta)}\left[e^{-2 \delta t}-e^{-2 \theta t}\right] \\
& \quad \leq\left(1+\frac{\lambda_{0} \gamma_{0}}{2(\theta-\delta)}\right) e^{-2 \delta t} V(0)-\frac{\lambda_{0} \gamma_{0}}{2(\theta-\delta)} V(0) e^{-2 \theta t}
\end{aligned}
$$

From (4.6) it follows that

$$
\begin{aligned}
E(t) \leq\left(1+\frac{\lambda_{0} \gamma_{0}}{2(\theta-\delta)}\right) & (1+\mu L) e^{-2 \delta t} E(0) \\
& -\frac{\lambda_{0} \gamma_{0}}{2(\theta-\delta)} E(0) e^{-2 \theta t}
\end{aligned}
$$

which implies (3.9).

In order to end the proof of Theorem 1, we need to prove that (4.16) holds. On the one hand, for $t=0$, inequality (4.16) holds by hypothesis in Theorem 1 , so that $E(0)<$ $\frac{1}{\Gamma}$. On the other hand, let (4.16) be false for some $t>0$ and let $t^{*}$ be the smallest instant such that $E\left(t^{*}\right) \geq R^{2}$. Since $E$ is continuous in time, we have $E\left(t^{*}\right)=R^{2}$ and $E(t)<R^{2}$ for $t \in\left[0, t^{*}\right)$. Therefore, the feasibility of inequality (3.5) and LMI (3.6) guarantee that (4.17) is true for all $t \in\left[0, t^{*}\right)$. Hence, by continuity,

$$
\begin{aligned}
E(t) & \leq\left(1+\frac{\lambda_{0} \gamma_{0}}{2 \theta-2 \delta}\right)(1+\mu L) e^{-2 \delta t} E(0) \\
& \leq\left(1+\frac{\lambda_{0} \gamma_{0}}{2 \theta-2 \delta}\right)(1+\mu L) \frac{1}{\Gamma}, \quad \forall t \in\left[0, t^{*}\right]
\end{aligned}
$$

The above inequality, together with the assumption (3.7), implies

$$
E(t) \leq\left(1+\frac{\lambda_{0} \gamma_{0}}{2 \theta-2 \delta}\right)(1+\mu L) \frac{1}{\Gamma}<R^{2}
$$

for all $t \in\left[0, t^{*}\right]$, which contradicts the definition of $t^{*}$. Therefore, (4.16) holds.

Note that the feasibility of the strict LMI (3.6) with $\delta=0$ implies its feasibility with a slightly larger $\delta_{0}>0$. Therefore, if the strict LMI (3.6) holds for $\delta=0$, then the closed-loop system is exponentially stable with a small decay rate.

Remark 4. It must be stressed that the present Lyapunov function cannot work for the case of a simple sampled-data control under averaged measurement and that the event-triggered law is critical in the proof of stability.

Remark 5. Given $K>\lambda+\delta$, the LMI conditions of Theorem 1 are always feasible for small enough $\gamma, \gamma_{0}$, $\Delta$ and large enough $\lambda_{0}$ such that $\lambda_{0} \gamma$ is small. By Schur complement, $\Phi \prec 0$ is equivalent to (4.14) with $x=L$. The latter holds for $\mu=\lambda_{1}=\gamma=\Delta=0$, and large 
enough $\lambda_{0}$. Thus, LMIs hold for small enough $\mu, \lambda_{1}, \gamma$, $\Delta, \gamma_{0}, R$ with appropriate (large enough) $\lambda_{0}$.

Remark 6. Let us explain here what prevents us from obtaining such results under point measurements. For the case of averaged measurements, in the proof of Theorem 1, we need the Lyapunov functional to be continuous in $L^{2}$-norm. For the case of point measurements, for a matter of continuity in the space variable, we need to guarantee that the Lyapunov functional is continuous in $H^{1}$-norm. But this requires that the solution is in $C\left([0, T] ; H^{1}(0, L)\right)$, therefore requiring more regular initial and boundary data than it is the case here.

\section{Extension to the controller distributed on subdomains}

In this subsection, we are concerned with the case that the actuation does not cover the whole domain $\Omega$ and the averaged measurements are measured over the parts of the subdomains. As in [28], let

$$
\begin{gathered}
0 \leq \tilde{x}_{1}<\tilde{x}_{2} \leq \tilde{x}_{3}<\tilde{x}_{4} \leq \cdots \leq \tilde{x}_{2 N-1}<\tilde{x}_{2 N} \leq L, \\
{\left[\tilde{x}_{2 j-1}, \tilde{x}_{2 j}\right] \subset\left[x_{j-1}, x_{j}\right], j=1,2, \cdots, N .}
\end{gathered}
$$

Denote $\widetilde{\Omega}_{j} \triangleq\left[\tilde{x}_{2 j-1}, \tilde{x}_{2 j}\right]$. Now we study the system (2.1) under the event-triggered controller

$$
f(x, t)=-K \sum_{j=1}^{N} \tilde{z}_{j}\left(t_{k}\right) \mathbb{1}_{\widetilde{\Omega}_{j}}(x), \quad K>\lambda,
$$

where

$$
\tilde{z}_{j}\left(t_{k}\right)=\frac{1}{\left|\widetilde{\Omega}_{j}\right|} \int_{\widetilde{\Omega}_{j}} z\left(x, t_{k}\right) d x,\left|\widetilde{\Omega}_{j}\right|=\tilde{x}_{2 j}-\tilde{x}_{2 j-1} .
$$

By applying the first mean value theorem, since $z \in$ $C\left([0, T], L^{2}(0, L)\right)$ we obtain that there exists a point $\bar{x}_{t}^{j} \in \widetilde{\Omega}_{j}$ such that

$$
\frac{1}{\left|\widetilde{\Omega}_{j}\right|} \int_{\widetilde{\Omega}_{j}} z(x, t) d x=z\left(\bar{x}_{t}^{j}, t\right)
$$

Then the controller (5.1) can be rewritten as

$$
f(x, t)=-K \sum_{j=1}^{N}\left[z\left(\bar{x}_{t}^{j}, t\right)-\tilde{\rho}_{j}(t)\right] \mathbb{1}_{\widetilde{\Omega}_{j}}(x),
$$

where $\tilde{\rho}_{j}(t)=\frac{1}{\left|\widetilde{\Omega}_{j}\right|} \int_{\widetilde{\Omega}_{j}} e_{k}(x, t) d x$.

This leads to the closed-loop system

$$
\left\{\begin{array}{c}
\partial_{t} z+z \partial_{x} z+\partial_{x} z+\nu \partial_{x x x} z-\lambda z \\
=-K \sum_{j=1}^{N}\left[z\left(\bar{x}_{t}^{j}, t\right)-\tilde{\rho}_{j}(t)\right] \mathbb{1}_{\widetilde{\Omega}_{j}}(x), \\
\operatorname{in}(0, L) \times\left[t_{k}, t_{k+1}\right), \quad k \in \mathbb{N}, \\
z(0, t)=z(L, t)=0, \partial_{x} z(L, t)=0, \quad \forall t \geq 0, \\
z(x, 0)=z^{0}(x), \quad \forall x \in(0, L) .
\end{array}\right.
$$

Denote

$$
l_{j} \triangleq \max \left\{\tilde{x}_{2 j}-x_{j-1}, x_{j}-\tilde{x}_{2 j-1}\right\} .
$$

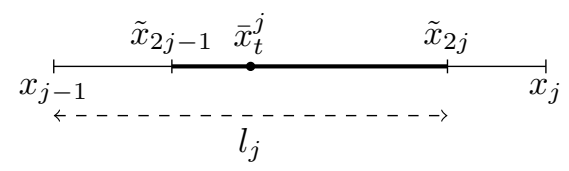

Fig. 1. Subdomain $\widetilde{\Omega}_{j}=\left[\tilde{x}_{2 j-1}, \tilde{x}_{2 j}\right]$, point $\bar{x}_{t}^{j}$ and $l_{j}$

Then we have the following result:

Proposition 2. Consider the closed-loop system (5.5). Let $L>0, T>0$. Denote

$$
l \triangleq \max _{j} l_{j}, \bar{\Delta} \triangleq \min _{j} \frac{\left|\widetilde{\Omega}_{j}\right|}{\left|\Omega_{j}\right|}
$$

Given a desired decay rate $\delta>0$, a control gain $K>$ $\lambda+\delta$, length bounds $l>0, \bar{\Delta}>0$, and positive tuning parameters $\lambda_{0}, R, \theta>\delta, \gamma_{0}>0$, assume that there exist positive scalars $\mu, \lambda_{1}, \beta_{i}(i=1,2), \gamma$, and $\Gamma$ that solve the following optimization problem:

$$
\min \Gamma \quad \text { subject to }
$$

$$
\Phi=\left[\begin{array}{cccc}
-3 \mu \nu+\lambda_{1}+\beta_{2}+\frac{2}{3} \mu R L \sqrt{L}<0, & K(1+\mu L) \\
\phi_{11} & \beta_{2} \frac{\pi^{2}}{4 l^{2}} & 0 & \pi^{2} \\
* & -2 K \bar{\Delta}-\beta_{2} \frac{-K \mu L}{4 l^{2}} & 0 \\
* & * & -\beta_{1} & 0 \\
* & * & -\lambda_{0}
\end{array}\right] \prec 0
$$

where

$\phi_{11}=\mu+\lambda_{0} \gamma+(2 \lambda+2 \delta)(1+\mu L)+\beta_{1}-\beta_{2} \frac{\pi^{2}}{4 l^{2}}-\lambda_{1} \frac{\pi^{2}}{L^{2}}$. 
Then for any initial function $z^{0} \in L^{2}(0, L)$ satisfying $\left\|z^{0}\right\|_{L^{2}(0, L)}<\frac{1}{\sqrt{\Gamma}}$, the closed-loop system (5.5) under the event-triggering mechanism (3.4) is exponentially stable in the sense that (3.9) holds. Moreover, if the above LMIs hold with $\delta=0$, then the closed-loop system is exponentially stable with a small enough decay rate.

Proof. Consider $V(t)$ given by (4.5). Differentiating $V(t)$ along (5.5), for any $\lambda_{0}>0$ one gets

$$
\begin{aligned}
\dot{V}(t) & \leq \dot{V}(t)+\lambda_{0}\left[\gamma E(t)+\gamma_{0} E(0) e^{-2 \theta t}-\left\|e_{k}(\cdot, t)\right\|_{L^{2}(0, L)}^{2}\right] \\
& \leq-\nu\left|\partial_{x} z(0, t)\right|^{2}-3 \mu \nu \int_{0}^{L}\left|\partial_{x} z(x, t)\right|^{2} d x \\
& +\left(\mu+\lambda_{0} \gamma\right) \int_{0}^{L}|z(x, t)|^{2} d x+\frac{2}{3} \mu \int_{0}^{L} z^{3}(x, t) d x \\
& +2 \lambda \int_{0}^{L}(1+\mu L)|z(x, t)|^{2} d x-2 K \sum_{j=1}^{N} z^{2}\left(\bar{x}_{t}^{j}, t\right)\left|\widetilde{\Omega}_{j}\right| \\
& -2 K \mu \sum_{j=1}^{N} \int_{\Omega_{j}} x \mathbb{1}_{\widetilde{\Omega}_{j}}(x) z(x, t) z\left(\bar{x}_{t}^{j}, t\right) d x \\
& +2 K \sum_{j=1}^{N} \int_{\Omega_{j}} \mathbb{1}_{\widetilde{\Omega}_{j}}(x)(1+\mu x) z(x, t) \tilde{\rho}_{j}(t) d x \\
& +\lambda_{0} \gamma_{0} E(0) e^{-2 \theta t}-\lambda_{0}\left\|e_{k}(\cdot, t)\right\|_{L^{2}(0, L)}^{2} .
\end{aligned}
$$

Cauchy-Schwarz's inequality yields

$$
\begin{aligned}
& \int_{\Omega_{j}}\left[\mathbb{1}_{\widetilde{\Omega}_{j}}(x) \tilde{\rho}_{j}(t)\right]^{2} d x=\left|\widetilde{\Omega}_{j}\right| \tilde{\rho}_{j}^{2}(t)=\frac{1}{\left|\widetilde{\Omega}_{j}\right|}\left[\int_{\widetilde{\Omega}_{j}} e_{k}(x, t) d x\right]^{2} \\
& \leq \int_{\widetilde{\Omega}_{j}}\left|e_{k}(x, t)\right|^{2} d x \leq \int_{\Omega_{j}}\left|e_{k}(x, t)\right|^{2} d x
\end{aligned}
$$

so that

$$
\lambda_{0} \sum_{j=1}^{N} \int_{\Omega_{j}}\left[\left|e_{k}(x, t)\right|^{2}-\left[\mathbb{1}_{\widetilde{\Omega}_{j}}(x) \tilde{\rho}_{j}(t)\right]^{2}\right] d x \geq 0 .
$$

From $\widetilde{\Omega}_{j} \subset \Omega_{j}$, one has $\beta_{1}>0$ such that

$$
\beta_{1} \sum_{j=1}^{N} \int_{\Omega_{j}}\left[|z(x, t)|^{2}-\left[\mathbb{1}_{\widetilde{\Omega}_{j}}(x) z(x, t)\right]^{2}\right] d x \geq 0
$$

Wirtinger's inequality leads to (4.9) and

$$
\begin{aligned}
& \int_{\Omega_{j}}\left[z(x, t)-z\left(\bar{x}_{t}^{j}, t\right)\right]^{2} d x \\
& =\int_{x_{j-1}}^{\bar{x}_{t}^{j}}\left[z(x, t)-z\left(\bar{x}_{t}^{j}, t\right)\right]^{2} d x+\int_{\bar{x}_{t}^{j}}^{x_{j}}\left[z(x, t)-z\left(\bar{x}_{t}^{j}, t\right)\right]^{2} d x \\
& \leq \frac{4\left(\bar{x}_{t}^{j}-x_{j-1}\right)^{2}}{\pi^{2}} \int_{x_{j-1}}^{\bar{x}_{t}^{j}}\left|\partial_{x} z(x, t)\right|^{2} d x+\frac{4\left(x_{j}-\bar{x}_{t}^{j}\right)^{2}}{\pi^{2}} \int_{\bar{x}_{t}^{j}}^{x_{j}}\left|\partial_{x} z(x, t)\right|^{2} d x .
\end{aligned}
$$

From (5.7) and (5.13), it follows that

$$
\int_{\Omega_{j}}\left[z(x, t)-z\left(\bar{x}_{t}^{j}, t\right)\right]^{2} d x \leq \frac{4 l^{2}}{\pi^{2}} \int_{\Omega_{j}}\left|\partial_{x} z(x, t)\right|^{2} d x
$$

which implies

$\beta_{2} \sum_{j=1}^{N} \int_{\Omega_{j}}\left[\left|\partial_{x} z(x, t)\right|^{2}-\frac{\pi^{2}}{4 l^{2}}\left[z(x, t)-z\left(\bar{x}_{t}^{j}, t\right)\right]^{2}\right] d x \geq 0$

for some constant $\beta_{2}>0$.

Set $\tilde{\eta}(x, t)=\left\{z(x, t), z\left(\bar{x}_{t}^{j}, t\right), \mathbb{1}_{\widetilde{\Omega}_{j}}(x) z(x, t), \mathbb{1}_{\widetilde{\Omega}_{j}}(x) \rho_{j}(t)\right\}$.

Using (4.8), (4.9), (5.7), (5.12) and applying Sprocedure, we have

$$
\begin{aligned}
& \dot{V}(t)+2 \delta V(t) \\
& \leq \dot{V}(t)+2 \delta V(t)+\lambda_{0}\left[\gamma E(t)+\gamma_{0} E(0) e^{-2 \theta t}-\left\|e_{k}(\cdot, t)\right\|_{L^{2}(0, L)}^{2}\right] \\
& +\lambda_{1}\left[\left\|\partial_{x} z(\cdot, t)\right\|_{L^{2}(0, L)}^{2}-\frac{\pi^{2}}{L^{2}}\|z(\cdot, t)\|_{L^{2}(0, L)}^{2}\right] \\
& +\lambda_{0} \sum_{j=1}^{N} \int_{\Omega_{j}}\left[\left|e_{k}(x, t)\right|^{2}-\left[\mathbb{1}_{\tilde{\Omega}_{j}}(x) \rho_{j}(t)\right]^{2}\right] d x \\
& +\beta_{1} \sum_{j=1}^{N} \int_{\Omega_{j}}\left[|z(x, t)|^{2}-\left[\mathbb{1}_{\tilde{\Omega}_{j}}(x) z(x, t)\right]^{2}\right] d x \\
& +\beta_{2} \sum_{j=1}^{N} \int_{\Omega_{j}}\left[\left|\partial_{x} z(x, t)\right|^{2}-\frac{\pi^{2}}{4 l^{2}}\left[z(x, t)-z\left(\bar{x}_{t}^{j}, t\right)\right]^{2}\right] d x \\
& \leq \sum_{j=1}^{N} \int_{\Omega_{j}} \tilde{\eta}(x, t)^{\top} \tilde{\Phi}(x) \tilde{\eta}(x, t)+\lambda_{0} \gamma_{0} E(0) e^{-2 \theta t} \\
& -\left(3 \mu \nu-\lambda_{1}-\beta_{2}-\frac{2 \mu}{3} L \sqrt{L}\|z(\cdot, t)\|_{L^{2}(0, L)}\right)\left\|\partial_{x} z(\cdot, t)\right\|_{L^{2}(0, L)}^{2},
\end{aligned}
$$

where

$$
\tilde{\Phi}(x)=\left[\begin{array}{cccc}
\phi_{11} & \beta_{2} \frac{\pi^{2}}{4 l^{2}} & 0 & K(1+\mu x) \\
* & -2 K \bar{\Delta}-\beta_{2} \frac{\pi^{2}}{4 l^{2}} & -K \mu x & 0 \\
* & * & -\beta_{1} & 0 \\
* & * & * & -\lambda_{0}
\end{array}\right],
$$

with $\phi_{11}$ as in (5.11). Thus, by Schur complement, the LMIs (5.8), (5.9) yield (3.9). 
Remark 7. Given $\bar{\Delta}<1$ and $K>\frac{\lambda+\delta}{\bar{\Delta}}$, the LMI conditions of Proposition 2 are always feasible for small enough $\gamma, \gamma_{0}, R<\frac{9 \nu}{2 L \sqrt{L}}$, and large enough $\lambda_{0}$. By Schur complement, $\tilde{\Phi} \prec 0 \Longleftrightarrow \phi_{11}+\lambda_{0}^{-1} K^{2}(1+\mu L)^{2}$

$-\left(\beta_{2} \frac{\pi^{2}}{4 l^{2}}\right)^{2}\left[-2 K \bar{\Delta}-\beta_{2} \frac{\pi^{2}}{4 l^{2}}+\beta_{1}^{-1} K^{2} \mu^{2} L^{2}\right]^{-1}<0$. Choose $\beta_{2}=2 K \bar{\Delta} \frac{4 l^{2}}{\pi^{2}}$. The latter holds for $\mu=\lambda_{1}=\gamma=$ 0 , small enough $\beta_{1}$ and large enough $\lambda_{0}$. Thus, LMIs hold for small enough $\mu, \lambda_{1}, \beta_{1}, \gamma, \gamma_{0}, R<\frac{9 \nu}{2 L \sqrt{L}}$ with appropriate (large enough) $\lambda_{0}$, (small enough) $l$ such that $\lambda_{0} \gamma$ and $2 K \bar{\Delta} \frac{4 l^{2}}{\pi^{2}}$ are small.

\section{$6 \quad$ Numerical examples}

Consider the KdV system:

$$
\left\{\begin{array}{l}
\partial_{t} z+z \partial_{x} z+\partial_{x} z+\nu \partial_{x x x} z-\lambda z=f(x, t) \\
\quad \forall 0<x<L, t \geq 0 \\
z(0, t)=z(L, t)=\partial_{x} z(L, t)=0, \\
z(x, 0)=z^{0}(x)=0.32\left(1-\cos \left(\frac{2 \pi x}{L}\right)\right), x \in[0, L]
\end{array}\right.
$$

where $\nu>0$ will be chosen below.

We will give simulation for the following cases:

- Open-loop system without input (i.e. $f(x, t)=0$ )

- Closed-loop system under continuous-time controller $f(x, t)=-K z(x, t)$

- Closed-loop system under event-triggered controller distributed on the whole domain with averaging $f(x, t)=-K \sum_{j=1}^{N} \bar{z}_{j}\left(x, t_{k}\right) \mathbb{1}_{\Omega_{j}}(x)$

- Closed-loop system under event-triggered controller distributed on subdomains with averaging $f(x, t)=$ $-K \sum_{j=1}^{N} \tilde{z}_{j}\left(x, t_{k}\right) \mathbb{1}_{\tilde{\Omega}_{j}}(x)$

where $K>\lambda$ is a controller gain.

Example 1: For the event-triggered control law (2.3) under averaged measurements, we verify LMI conditions of Theorem 1 with $K=L=1, \lambda=0.5, \nu=0.3, \delta=0.4$, $\Delta=0.1, R=0.5$. We find that the closed-loop system under event-triggering mechanism (3.4) with $\theta=$ $2, \gamma=0.00029$ and $\gamma_{0}=0.02$ is exponentially stable for $\mu=0.5401$ and for any initial values satisfying $\left\|z^{0}\right\|_{L^{2}(0,1)}<\frac{1}{\sqrt{6.1997}} \approx 0.4$.

A finite difference method is used to illustrate the effect of the proposed event-triggered control law. The steps of space and time are chosen as 0.05 and 0.0001 , respectively. Fig. 2 illustrates the evolution of the state of the

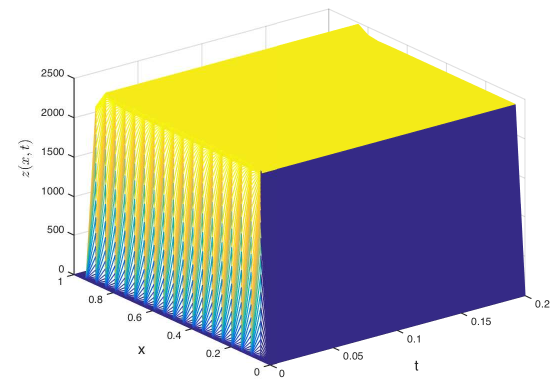

Fig. 2. State of the open-loop system with $\lambda=0.5$

open-loop KdV system. It is seen that the open-loop system is unstable.

Fig. 3 illustrates the evolution of the state of the closed-loop $\mathrm{KdV}$ system under the event-triggering mechanism

$$
\begin{aligned}
t_{k+1}= & \inf \left\{t \geq t_{k} \mid\right. \\
& \left.\left\|e_{k}\right\|_{L^{2}(0, L)}^{2} \geq 0.00029 E(t)+0.02 E(0) e^{-4 t}\right\} .
\end{aligned}
$$

with the control law (2.3) where $\bar{z}_{j}\left(t_{k}\right)=10 \int_{\Omega_{j}} z\left(\zeta, t_{k}\right) d \zeta$, $t \in\left[t_{k}, t_{k+1}\right)$ subject to $x_{j}-x_{j-1}=\left|\Omega_{j}\right|=\Delta=0.1$. It shows that the state of closed-loop KdV system under the event-triggered controller converges exponentially to zero. Fig. 4 shows that the release time and release interval by event-triggering for $t \in[0,0.2]$.

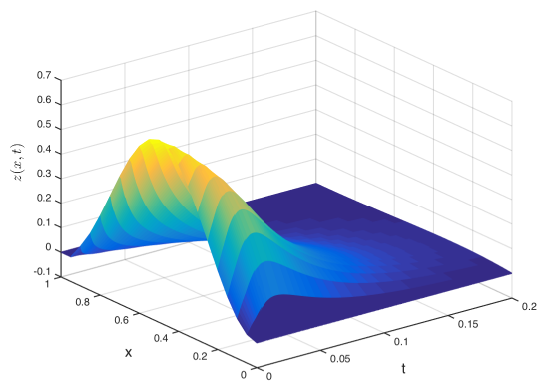

Fig. 3. State of the closed-loop system with the event-triggered control law (2.3) distributed over all domain

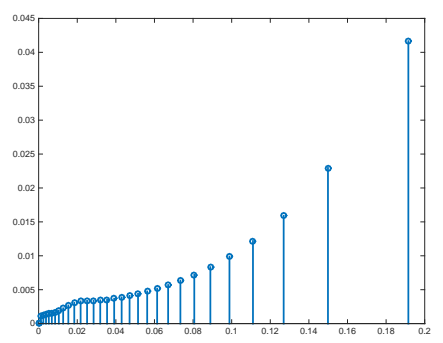

Fig. 4. Release instants and release interval by event-triggering 
Fig. 5 demonstrates the time evolution of $\ln (E(t))$ for the open-loop system, the closed-loop system under continuous-time controller, and the closed-loop system under the event-triggered controller. The simulations show that the event-triggered controller improves the performance.

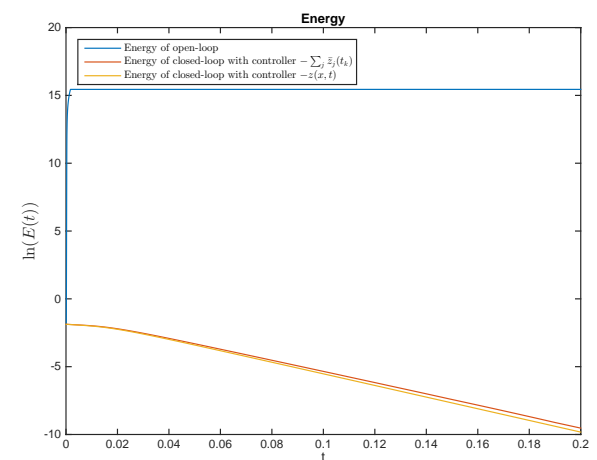

Fig. 5. $\ln (E(t))$ of the open-loop system, closed-loop system under continuous-time/event-triggered controller

Example 2: For the event-triggered control law (5.1) under averaged and localized measurements, we verify LMI conditions of Proposition 2 with $K=L=1, \lambda=$ $0.5, \lambda_{0}=1, \nu=0.3, \delta=0.4, R=0.5, l=0.2, \bar{\Delta}=$ $1 / 3$. We find that the closed-loop system under eventtriggering mechanism (3.4) with $\theta=2, \gamma=0.0013$ and $\gamma_{0}=0.02$ is exponentially stable for $\mu=0.0235$ and for any initial values satisfying $\left\|z^{0}\right\|_{L^{2}(0,1)}<\frac{1}{\sqrt{4.5603}} \approx$ 0.46. We proceed further with numerical simulations of the closed-loop KdV system under the event-triggering mechanism

$$
\begin{aligned}
t_{k+1}= & \inf \left\{t \geq t_{k} \mid\right. \\
& \left.\left\|e_{k}\right\|_{L^{2}(0, L)}^{2} \geq 0.0013 E(t)+0.02 E(0) e^{-4 t}\right\} .
\end{aligned}
$$

Let $x_{0}=0, x_{1}=0.3, x_{2}=0.6, x_{3}=0.9$ and $x_{4}=1$. Set $\tilde{x}_{1}=0.1, \tilde{x}_{2}=0.2, \tilde{x}_{3}=0.4, \tilde{x}_{4}=0.5, \tilde{x}_{5}=0.7$, $\tilde{x}_{6}=0.8, \tilde{x}_{7}=0.9, \tilde{x}_{8}=1$. The simulations show that the state of closed-loop $\mathrm{KdV}$ system converges to zero (see Fig. 6).

\section{Conclusion}

The present work discusses event-triggered control of the nonlinear KdV equation. An event-triggering mechanism has been proposed to reduce the number of control update. By constructing an appropriate Lyapunov functional, sufficient LMI-based conditions have been investigated while ensuring that the closed-loop system is regionally exponentially stable. The avoidance of Zeno behaviour is guaranteed. The presented method gives efficient tools for various event-triggered controller and observer design problems for nonlinear PDEs.

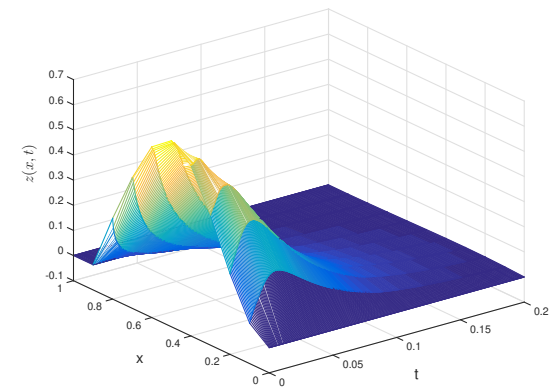

Fig. 6. State of the closed-loop system with the event-triggered control law (5.1) distributed over subdomains

\section{Appendix A}

Lemma A.3 (Sobolev embedding and inequality). The embedding $H^{1}(0, L) \subset C([0, L])$ is compact and for any $g \in H_{0}^{1}(0, L)$, it holds $\|g\|_{L^{\infty}(0, L)} \leq \sqrt{L}\left\|g^{\prime}\right\|_{L^{2}(0, L)}$.

Lemma A.4 (Wirtinger inequality [12]). Assume that $g \in H^{1}(0, L)$ with $g(0)=0$ or $g(L)=0$. Then $\|g\|_{L^{2}(0, L)}^{2} \leq \frac{4 L^{2}}{\pi^{2}}\left\|g^{\prime}\right\|_{L^{2}(0, L)}^{2}$. Moreover, if $g \in H_{0}^{1}(0, L)$, then $\|g\|_{L^{2}(0, L)}^{2} \leq \frac{L^{2}}{\pi^{2}}\left\|g^{\prime}\right\|_{L^{2}(0, L)}^{2}$.

Lemma A.5 (Poincaré inequality $[10,12])$. Assume that $g \in H^{1}(0, L)$ with $\int_{0}^{L} g(x) d x=0$. Then $\|g\|_{L^{2}(0, L)}^{2} \leq$ $\frac{L^{2}}{\pi^{2}}\left\|g^{\prime}\right\|_{L^{2}(0, L)}^{2}$.

\section{References}

[1] Azouani, A., Titi, E.S. (2014) Feedback control of nonlinear dissipative systems by finite determining parameters A reaction-diffusion Paradigm. Evolution Equations and Control Theory, 3, pp. 579-594.

[2] Baudouin, L., Crépeau, E., Valein, J. (2019) Two approaches for the stabilization of nonlinear $\mathrm{KdV}$ equation with boundary time-delay feedback. IEEE Transactions on Automatic Control., 64, pp. 1403-1414.

[3] Cerpa, E. (2014) Control of a Korteweg-de Vries equation: a tutorial. Math. Control Relat. Fields, 4, pp. 45-99.

[4] Cerpa, E., Coron, J.-M. (2013) Rapid stabilization for a Korteweg-de Vries equation from the left Dirichlet boundary condition. IEEE Transactions on Automatic Control. 58, pp. 1688-1695.

[5] Chapouly, M. (2009) Global controllability of a nonlinear Korteweg-de Vries equation. Commun. Contemp. Math., 11, pp. 495-521.

[6] Coron, J.M. (2007) Control and Nonlinearity. American Mathematical Society.

[7] Espitia, N., Karafyllis, I., Krstic, M. (2019) Event-triggered boundary control of constant-parameter reaction-diffusion PDEs: a small-gain approach, arXiv:1909.10472.

[8] Espitia, N., Tanwani, A., Tarbouriech, S. (2017) Stabilization of boundary controlled hyperbolic PDEs via Lyapunovbased event triggered sampling and quantization, in IEEE Conference on Decision and Control, Austria, pp. 1266-1271.

[9] Fridman, E., Blighovsky, A. (2012) Robust sampled-data control of a class of semilinear parabolic systems. Automatica, 48 , pp. $826-836$. 
[10] Fridman, E., Bar Am, N. (2013) Sampled-Data Distributed $H_{\infty}$ Control of Transport Reaction Systems. SIAM Journal on Control and Optimization, 51, pp. 1500-1527.

[11] Fridman, E. (2014) Introduction to time-delay systems: Analysis and control. Basel: Birkhäuser.

[12] Hardy, G. H., Littlewood, J. E., Pólya, G. (1988). Inequalities. Mathematical Library, Cambridge.

[13] Heemels, W.P.M.H, Donkers, M.C.F., Teel, A.R. (2013) Periodic event-triggered control for linear systems. IEEE Transactions on Automatic Control, 58, pp. 847-861.

[14] Kang, W., Fridman, E. Distributed stabilization of Kortewegde Vries-Burgers equation in the presence of input delay. Automatica, 100 (2019), pp. 260-273.

[15] Lunasin, E., Titi, E.S. (2017) Finite determining parameters feedback control for distributed nonlinear dissipative systems - a computational study. Evolution Equations and Control Theory., 6, pp. 535-557.

[16] Marx, S., Cerpa, E. (2018) Output feedback stabilization of the Korteweg-de Vries equation. Automatica, 87, pp. 210217.

[17] Pazoto, A. F. (2005) Unique continuation and decay for the Korteweg-de Vries equation with localized damping, ESAIM Control Optim. Calc. Var., 11, pp. 473-486.

[18] Pazy, A. (1983). Semigroups of linear operators and applications to partial differential equations. New York: Springer-Verlag.

[19] Perla Menzala, G. , Vasconcellos, C. F. and Zuazua, E. (2002) Stabilization of the Korteweg-de Vries equation with localized damping, Quart. Appl. Math., 60, pp. 111-129.

[20] Rosier, L. (1997) Exact boundary controllability for the Korteweg-de Vries equation on a bounded domain. ESAIM Control Optim. Calc. Var., 2, pp. 33-55.

[21] Rosier, L., Bing-Yu, Z. (2009) Control and stabilization of the Korteweg-de Vries equation: recent progresses. Jrl Syst Sci $\&$ Complexity, 22, pp. 647-682.

[22] Selivanov, A., Fridman, E. (2016) Distributed event-triggered control of diffusion semilinear PDEs. Automatica, 68, pp. 344-351.

[23] Selivanov, A., Fridman, E. (2016) Event-triggered $H_{\infty}$ control: a switching approach. IEEE Transactions on Automatic Control, 61, pp. 3221-3226.

[24] Seuret, A., Prieur, C., Tarbouriech, S. and Zaccarian, L. (2016) LQ-based event-triggered controller co-design for saturated linear systems. Automatica, 74, pp. 47-54.

[25] Tabuada, P. (2007) Event-triggered real-time scheduling of stabilizing control tasks. IEEE Transactions on Automatic Control, 52, pp. 1680-1685.

[26] Tallapragada, P., Chopra, N. (2014) Decentralized eventtriggering for control of nonlinear systems. IEEE Transactions on Automatic Control, 59, pp. 3312-3324.

[27] Tang, S.X., Krstic, M. (2013) Stabilization of linearized Korteweg-de Vries Systems with anti-diffusion, in American control conference, USA, pp. 3302-3307.

[28] Wang, J. W., Wu, H. N. (2014) Lyapunov-based design of locally collocated controllers for semi-linear parabolic PDE systems. Journal of the Franklin Institute., 351, pp. 429-441. 